\title{
LC-high resolution MS in environmental analysis: from target screening to the identification of unknowns
}

\author{
Martin Krauss • Heinz Singer • Juliane Hollender
}

Received: 22 January 2010 /Revised: 19 February 2010 / Accepted: 21 February 2010 /Published online: 16 March 2010

(C) Springer-Verlag 2010

\begin{abstract}
This article provides an overview of the state-ofthe-art and future trends of the application of LC-high resolution mass spectrometry to the environmental analysis of polar micropollutants. Highly resolved and accurate hybrid tandem mass spectrometry such as quadrupole/timeof-flight and linear ion trap/orbitrap technology allows for a more reliable target analysis with reference standards, a screening for suspected analytes without reference standards, and a screening for unknowns. A reliable identification requires both high resolving power and high mass spectral accuracy to increase selectivity against the matrix background and for a correct molecular formula assignment to unknown compounds. For the identification and structure elucidation of unknown compounds within a reasonable time frame and with a reasonable soundness, advanced automated software solutions as well as improved prediction systems for theoretical fragmentation patterns, retention times, and ionization behavior are needed.
\end{abstract}

Keywords High resolution mass spectrometry · Mass accuracy · Polar organic compounds · Non-target screening · Resolving power

\section{Introduction}

LC-MS technologies have opened the analytical window to thermolabile, polar compounds within the last 15 years. As a result, polar organic micropollutants have moved increas-

M. Krauss $\cdot H$. Singer $(\bowtie) \cdot J$. Hollender

Eawag, Swiss Federal Institute of Aquatic Science and

Technology,

8600 Dübendorf, Switzerland

e-mail: heinz.singer@eawag.ch ingly into the focus of environmental scientists, regulatory agencies, and politicians. On the basis of a large number of studies, it became evident that many polar micropollutants such as pesticides, pharmaceuticals, and industrial chemicals are present in the environment. Additionally, transformation products might be produced, of which perhaps just a few have so far been identified (e.g., [1]). To meet the challenges posed when analyzing a mixture of many known and unknown compounds at low concentrations in complex matrices, a range of different LC-MS technologies have been put forward in recent years. In particular, the coupling of LC to high resolution mass spectrometry (HRMS) with high mass accuracy has emerged as a powerful tool. Among the possible ionization techniques electrospray (ESI) is by far the most widely used as compared with atmosphericpressure chemical ionization (APCI) or the more recent atmospheric-pressure photoionization (APPI).

A brief overview of commercially available mass spectrometers used with LC and their figures of merit are given in Table 1. Instruments with triple quadrupole (QqQ) and to a lesser extent quadrupole ion trap (QIT) technologies are the workhorses in target analysis. These usually offer high sensitivity and selectivity, but operate at unit resolution and in the case of QqQ have a low sensitivity in full-scan mode, which limits their capabilities in the detection of unknowns. LC-time-of-flight (TOF) instruments with a high acquisition speed are now generally capable of 20,000 resolving power ( $R$; defined throughout this article at full width at half maximum, FWHM), but sensitivity and the linear dynamic range are still lower than for the other technologies. Fourier transform ion cyclotron resonance (FT-ICR) mass analyzers have been rarely used in polar organic trace analytics due to their high costs, but the orbitrap technology, which was introduced to the market in 2005, is increasingly applied due to the 
Table 1 Comparison of commercial mass analyzers in LC-MS instruments. Typical values for an m/z range of 300-400 are given; specific instruments or configurations might achieve better figures of merit

\begin{tabular}{|c|c|c|c|c|}
\hline Mass spectrometer & $\begin{array}{l}\text { Resolving power } \\
\text { (FWHM) }\end{array}$ & Mass accuracy (ppm) & $\begin{array}{l}\text { Linear dynamic } \\
\text { range }\end{array}$ & Sensitivity $^{\mathrm{c}}$ (absolute mass) \\
\hline Triple quadrupole (QqQ) & Unit resolution $^{\mathrm{b}}$ & 50 & $10^{4}$ & Femto- to picogram (SRM) \\
\hline Quadrupole ion trap (QIT) & 10,000 & 50 & $10^{3}$ & Femto- to picogram (SRM, full scan) \\
\hline Time-of-flight (TOF) & 20,000 & 3 & $10^{2}-10^{3}$ & Picogram (full scan) \\
\hline Orbitrap & 100,000 & 2 & $10^{3}-10^{4}$ & Femto- to picogram (full scan) \\
\hline $\begin{array}{l}\text { Fourier transform ion cyclotron } \\
\text { resonance (FT-ICR) }\end{array}$ & $1,000,000$ & $\leq 1$ & $10^{4}$ & Picogram (full scan) \\
\hline
\end{tabular}

${ }^{\text {a }}$ Resolving power depends on $m / z$ range and scan speed on most instruments

${ }^{\mathrm{b}}$ Unit mass resolution is the resolution for standard quadrupole instruments; with special hyperbolic quadrupole instruments a resolving power of 5,000 and a mass accuracy of 5 ppm can be achieved without a major loss of signal intensity

${ }^{c}$ Sensitivity depends strongly on the ionization efficiency of the compound in the ion source

combination of high $R$ (up to 100,000), high mass accuracy $(<2 \mathrm{ppm})$, and a sensitivity down to the femtogram range. Combination of two different mass spectrometer types in so-called hybrid instruments such as quadrupole/TOF (QTOF) or linear ion trap/orbitrap (LTQ Orbitrap) have particularly shown excellent detection and identification capabilities for low molecular weight compounds in various matrices based on high resolution accurate mass measurement of precursor and product ions ([2-5]).
In the following discussion we illustrate the features, advantages, and limitations of LC coupled to HRMS for three conceptually different analytical approaches: (i) quantitative target analysis with reference standards; (ii) suspects screening without reference standards; and (iii) non-target screening of unknowns. A systematic workflow for all three approaches is depicted in Fig. 1, and their main characteristics and the state of the science are presented and discussed in the following sections. Finally, we highlight
Fig. 1 Comparison of systematic workflows for (i) quantitative target analysis with reference standards, (ii) suspects screening without reference standards, and (iii) non-target screening of unknowns in environmental samples by using LC-high resolution (tandem) mass spectrometry.

* Note that the $m / z$ range of the extraction window for the exact mass filtering depends on the mass accuracy and the resolving power of the mass spectrometer used

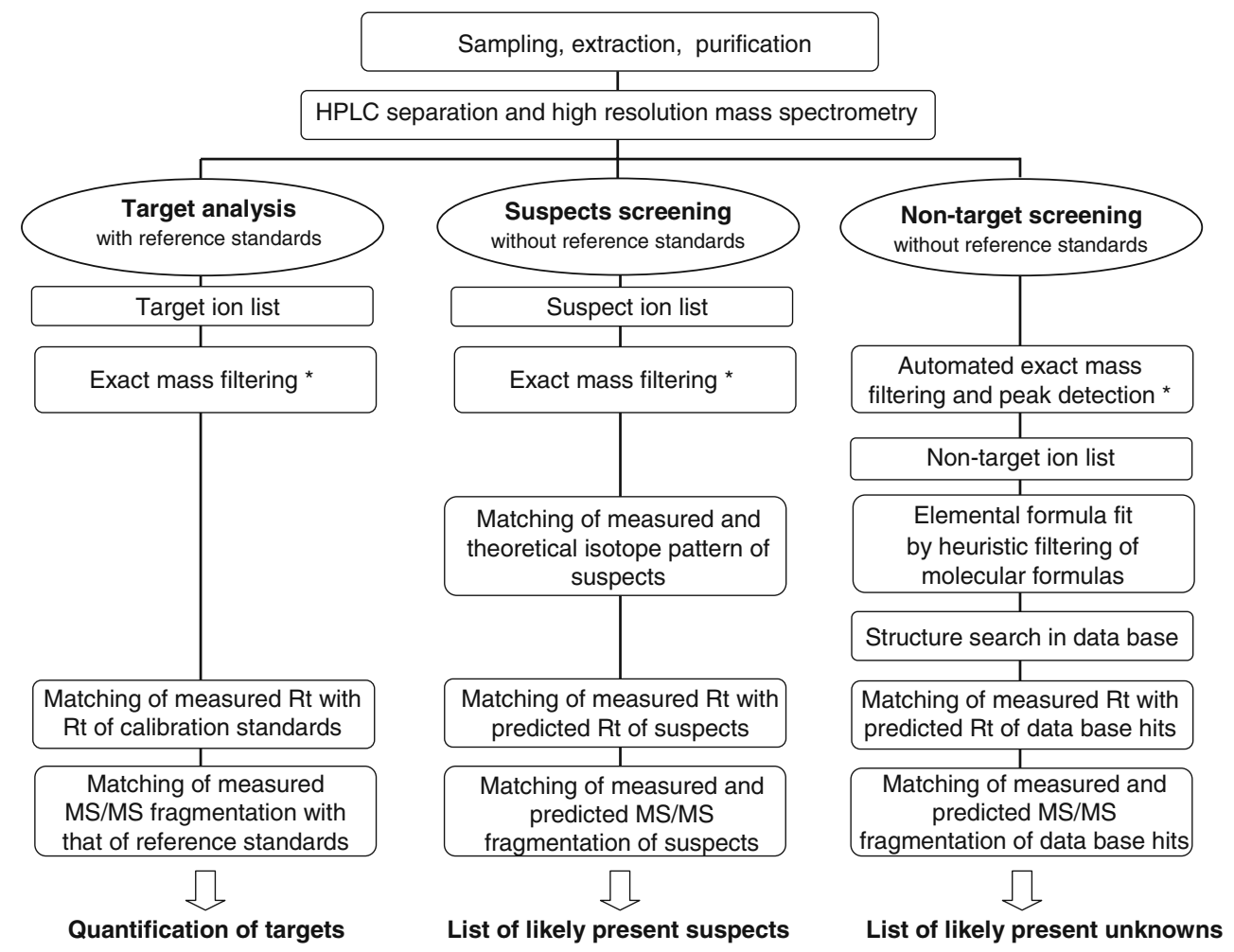


future trends and requirements. Although this article focuses on the HRMS detection and data evaluation steps, the preceding analytical procedures (extraction, enrichment/ purification, LC separation, ionization) are of equal importance for the different approaches. To gain optimum performance for the target, suspects, and non-target screening the conditions for extraction, purification, and LC separation have to be adapted for each workflow. A sophisticated HRMS approach cannot detect an analyte if it is not ionized by the chosen technique or if its ionization is suppressed by a strong matrix background due to insufficient cleanup.

\section{Target analysis}

The identification and quantification of micropollutants at low concentrations requires both a high sensitivity and selectivity against complex matrix backgrounds. For a large range of compounds, selected reaction monitoring (SRM) of precursor-product ion transitions by using QqQ or QIT instruments fulfills these prerequisites. A range of studies have shown, however, that monitoring only one transition might result in false positive identifications for individual compounds and thus at least two transitions are required [6]. While this approach has proved adequate for the majority of polar compounds in environmental matrices [6], several limitations have become evident:

(i) Under the constraints of at least two transitions, SRM methods are typically limited to about 100-150 target analytes depending on chromatographic separation, as otherwise accuracy or sensitivity deteriorate due to an insufficient temporal peak resolution or too short acquisition times for the individual MS/MS transitions, respectively.

(ii) For some analytes, only non-specific transitions such as the neutral loss of $\mathrm{H}_{2} \mathrm{O}$ or $\mathrm{CO}_{2}$ might occur, which are common also for matrix interferences.

(iii) Some analyte ions, particularly those of low molecular weight, show only one transition. In some cases only one product ion shows a sufficient signal intensity, while that of others is very low, resulting in higher limit of quantification (LOQ) values.

HRMS target analysis offers promising solutions to these three limitations of SRM analysis. Virtually all compounds present in a sample can be determined simultaneously with HRMS instruments operating in full-scan mode, making no preselection of compounds and associated SRM transitions necessary. Additionally, hybrid instruments offer the possibility of data-dependent (also termed informationdependent) MS/MS acquisition, i.e., an MS/MS analysis is triggered if a compound from a target ion list is detected in the full scan $[7,8]$. This additionally allows for full-scan product ion spectra recording within the same run for 500 compounds or more. However, on QTOF instruments, these capabilities are impaired by the limited sensitivity, which is about 1-2 orders of magnitude lower than those of QqQ instruments in SRM mode, and the limited dynamic range which is about 10-fold below that of QqQs [2]. Thus, QTOF instruments have been used only occasionally for quantification (overviews given by $[9,10]$ ) and one established strategy is to use QqQ for quantification and a separate QTOF analytical run for confirmation of positive findings if concentrations are sufficiently high $[6,11]$. The LTQ Orbitrap instrument offers a better dynamic range and a sensitivity close to that of many QqQ instruments [12, 13], thus allowing for quantification and confirmation in a single analytical run.

Limitations (ii) and (iii) apply particularly to salt or solvent adduct ions, which are formed additionally to or instead of the protonated or deprotonated molecules upon ESI. While some alkali metal adducts often yield no product ions at all, other adducts dissociate unspecifically to yield the (de)protonated molecule upon SRM. An example is the analysis of the artificial sweetener sucralose, which was recently detected as a persistent compound in environmental water samples [14]. The formic acid adduct as well as the $[\mathrm{M}-\mathrm{H}]^{-}$ion show only unspecific SRM transitions (loss of formic acid and one chlorine atom, respectively). An HRMS monitoring of these ions, and the respective transitions provided a more selective confirmation of positive findings [15].

Figure 2 illustrates the superior performance of HRMS for target analysis as compared with SRM at unit mass resolution for low molecular weight $\mathrm{N}$-nitrosamines (74$199 \mathrm{Da}$ ) in wastewater samples by using an LTQ Orbitrap [12]. The number of intense and specific MS/MS transitions was small. Sensitivity in QqQ or QIT analyzers was usually limited by a high background in SRM experiments (Fig. 2a, left), which particularly increased with increasing complexity of the matrix, as can be seen by a comparison of secondary and primary sewage treatment plant effluent. An excellent selectivity regardless of the different matrices could be achieved for most compounds with an $R=30,000$ (Fig. 2a, right), by which the target analytes could be distinguished from interferences of the same nominal $\mathrm{m} / \mathrm{z}$ (Fig. 2b). However, with high resolution full scan, false positive identifications might also be possible; a coeluting and isobaric compound was detected for $N$-nitrosomorpholine in wastewater samples, which could only be distinguished by MS/MS [12] or separated by using an LC column of different polarity. This highlights the fact that even with HRMS the selectivity provided by a preceding LC separation is useful and that tandem MS capabilities are recommended for an adequate confirmation of positive findings in complex environmental matrices. 
Ion trap, unit mass resolution

$\mathrm{SRM} m / z 115.1 \rightarrow 69.1$

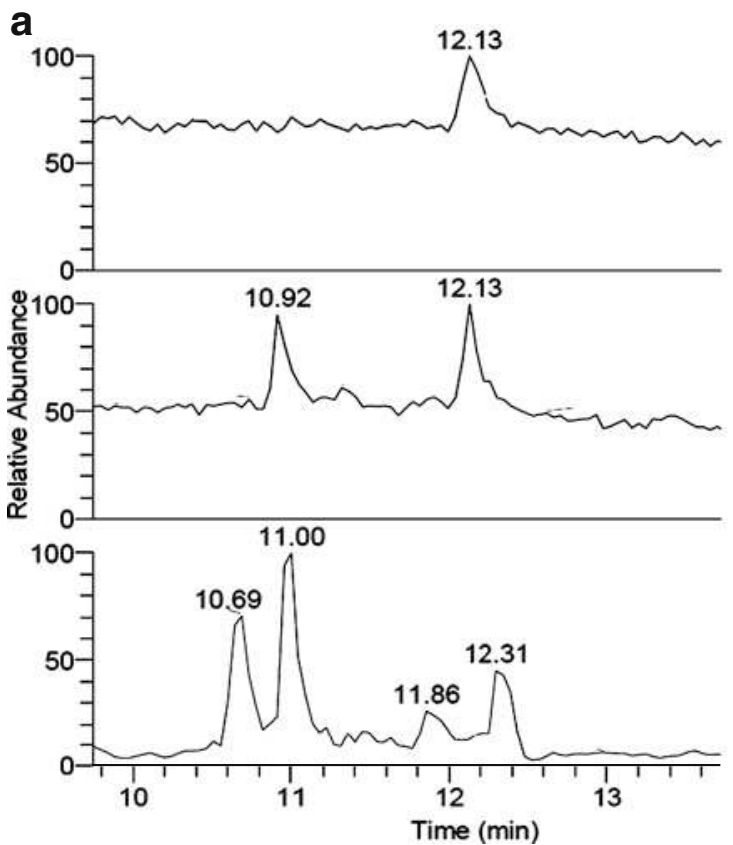

b

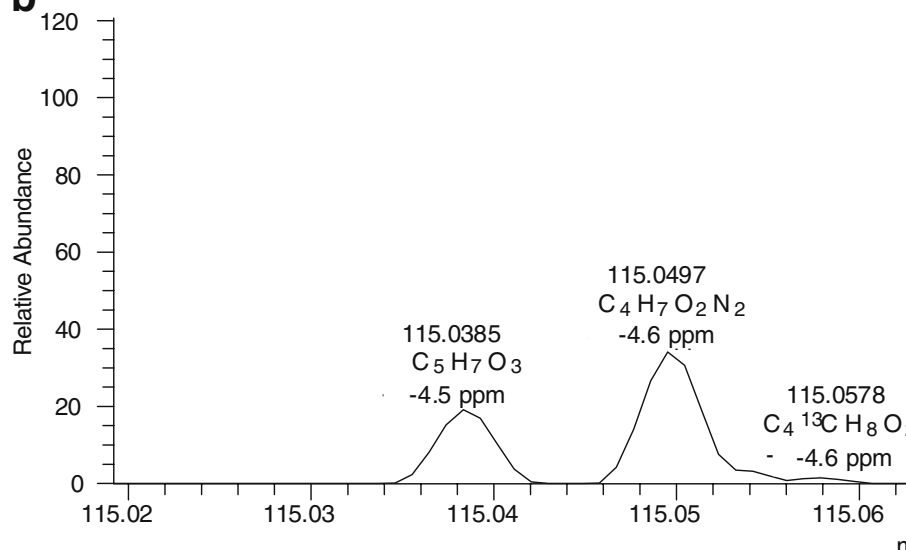

Orbitrap, $\mathrm{R}=\mathbf{3 0 , 0 0 0}$

full scan $\mathrm{m} / \mathrm{z} 115.0869 \pm 0.0012$

standard

spiked

secondary

effluent

spiked

primary

effluent
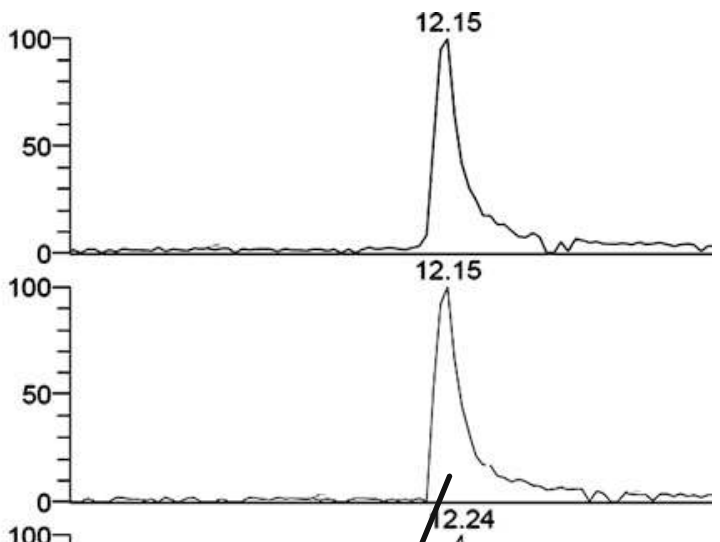

115.0692

$\mathrm{C}_{3}{ }^{13} \mathrm{CH}_{8} \mathrm{ON}_{3}$

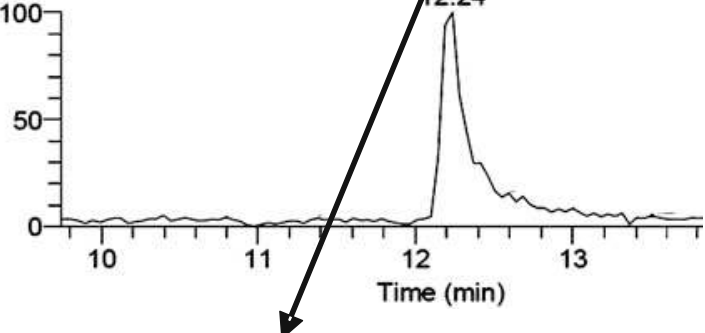

Time (min)

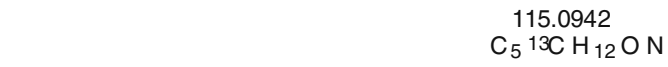

$\mathrm{C}_{5}{ }^{13} \mathrm{C} \mathrm{H}_{12} \mathrm{O}$

-4.4 ppm

$-3.1 \mathrm{ppm}$

$\mathrm{m} / \mathrm{z}$

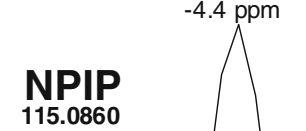

115.0860

-5.0 ppm
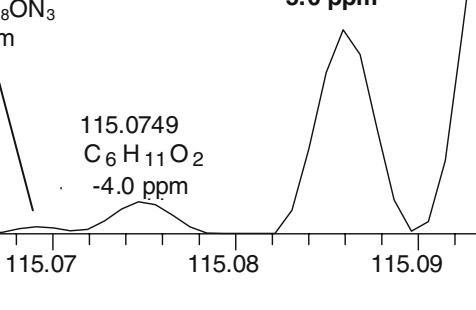

Fig. 2 a LC-MS chromatograms of $N$-nitrosopiperidine (NPIP) obtained from SRM at unit mass resolution (ion trap) and HRMS full scan at $R=30,000$ using an LTQ Orbitrap. A calibration standard of $10 \mathrm{ng} / \mathrm{mL}$ in water/methanol 95:5, and extracts of a secondary and a primary effluent sample from a sewage treatment plant both spiked at

\section{Suspects screening}

In contrast to target analysis, the suspects screening approach (Fig. 1) does not rely on reference standards for quantification and confirmation. These reference standards are currently not available for a large number of potential environmental contaminants, in particular transformation products. However, compound-specific information for suspects is available, such as molecular formula and structure, which can be efficiently used in the identification and confirmation process. First, the molecular formula
$20 \mathrm{ng} / \mathrm{L}$ are shown. All peaks correspond to about $200 \mathrm{pg}$ of compound on column. b Section of the high resolution full-scan mass spectrum from the secondary effluent sample extract showing NPIP and interfering compounds of the same nominal mass with molecular formula assignments and mass accuracy. Further analytical details are given in [12] allows for the calculation of an exact $\mathrm{m} / \mathrm{z}$ of the expected ion, which is in turn extracted from the high resolution fullscan chromatogram. For ESI, this task benefits from the fact that predominantly $[\mathrm{M}+\mathrm{H}]^{+}$and $[\mathrm{M}-\mathrm{H}]^{-}$ions are formed, except for some compounds which exclusively show adduct formation. In the case of positive findings, further confirmatory steps based solely on structure-derived information can be employed (Fig. 1), as exemplified by Kern et al. [16]. In their study, besides literature sources, a knowledge-based pathway prediction software tool was used to generate a list of 1,794 suspected microbial 
transformation products of polar micropollutants, which were searched for in surface water samples by using an LTQ Orbitrap at $R=60,000$. The large number of suspects required an efficient filtering approach, comprising rather straightforward and obvious criteria such as absence in analytical blanks and the match of the observed isotope pattern with the theoretically predicted ones for the molecular formula of the suspect. Furthermore, physicochemical properties and in turn the chromatographic retention times were predicted from the molecular structure. Such a quantitative approach has so far not been applied in environmental screening, but the wide prediction margin obtained in this study renders it more a soft plausibility check as compared with the isotope match criterion. Finally, Kern et al. [16] tentatively identified 19 transformation products which all later could be confirmed by using reference standards. This indicates that under the criteria employed the probability of false positive findings is low.

A less straightforward problem to assess is the possible occurrence of false negatives, as it is inherent in the suspects screening approach (as well as in the unknown screening approach, see below). Without an analytical standard it is not possible to prove from the outset whether a compound present in a sample will be identified in the chromatogram, as it could get lost during any step of the analytical procedure or is not ionized as anticipated. Thus, a careful validation of the whole procedure using a range of reference standards and a comparative assessment based on the (estimated) physicochemical properties of the suspects is a prerequisite to minimize the occurrence of false negatives. Another reason for false negatives is an insufficient mass resolution, as unresolved isobaric ions will yield an inaccurate "mixed" mass [17]. The resolving power actually needed depends strongly on the molecular composition as well as on the molecular weight of the compound and the interferences derived from the matrix background.

The impact of the matrix background is exemplified in Fig. 3 for soil extracts, in which pesticides and suspected transformation products were searched for. The distribution of ions from the natural soil matrix centers in a band from about $50-150 \mathrm{mDa}$ mass defect at $\mathrm{m} / z 150$ to $200-300 \mathrm{mDa}$ mass defect at $m / z 450$ (Fig. 3a). This mass defect range corresponds typically to constituents and fragments of natural organic molecules such as fatty acids, peptides, polyphenols, carbohydrates, and humic acids, and is dominated in its composition by $\mathrm{C}, \mathrm{H}, \mathrm{N}$, and $\mathrm{O}$. Obviously, a detection and confirmation of suspects falling into this "matrix band" might be difficult. This is shown for a suspected transformation product of the herbicide lenacil, which contains only $\mathrm{C}, \mathrm{H}, \mathrm{N}$, and $\mathrm{O}$. The extracted ion chromatogram of the $[\mathrm{M}+\mathrm{H}]^{+}$ion at $m / z 249.1234$ (i.e., mass defect of $123.4 \mathrm{mDa}$ ) shows a high background at retention times between 4 and $10 \mathrm{~min}$. In contrast, the extracted ion chromatogram of the herbicide linuron, which has a mass defect below this band $(19.2 \mathrm{mDa})$, shows one single peak without any interference from the matrix background over the whole chromatogram (Fig. 3b). A relatively low positive or a negative mass defect coincides with a low number of hydrogens and a relatively large number of heteroatoms $(\mathrm{Cl}, \mathrm{Br}, \mathrm{S}, \mathrm{F}, \mathrm{P})$. In the case of $\mathrm{Cl}$, $\mathrm{Br}$, and $\mathrm{S}$ these also show a prominent isotope pattern facilitating confirmation (see also Non-target screening). Similarly, compounds having a very high positive mass defect, caused by large aliphatic substructures and a low number of heteroatoms, do not fall into this "matrix band" as shown by $\mathrm{Li}$ and Brownawell [18] for quaternary ammonium compounds in water samples.

Thus, the identification of suspects with either high or low mass defects might generally require a lower $R$ than that of compounds predominantly composed of $\mathrm{C}, \mathrm{H}, \mathrm{O}$, and $\mathrm{N}$. Theoretical calculations show that for low molecular weight compounds a complete separation of the suspected peaks from a CHON background is also possible at a relatively low resolving power [12], as the number of chemically meaningful compositions is relatively low. This is indicated in Fig. 2b for $N$-nitrosopiperidine, which could be resolved from all other $\mathrm{CHON}$-containing ions as well as from some ${ }^{13} \mathrm{C}_{1}$-containing ions at $R=30,000$. With increasing $\mathrm{m} / \mathrm{z}$, the number of meaningful structural analogues increases dramatically [19]. While at $m / z=115$, 11 plausible CHON ions are possible, for an $\mathrm{m} / z$ of 249 this number increases to 87 (Fig. 3c), which can only be resolved completely at $R=200,000$. Luckily, only a very limited number of background ions seem to be actually present in mass spectra of environmental samples, as indicated in Fig. 3c. Thus, there is a good chance to resolve the suspect ions from the matrix at a lower $R$ than theoretically required, but there might always be an unfortunate case of a particular suspect ion that cannot be resolved from the matrix background. Beyond these theoretical considerations, practical experience up to now shows that a resolving power of 20,000 to 60,000 is required for many polar micropollutants, depending on the complexity of the matrix, which is corroborated from food residue analysis [3].

\section{Non-target screening}

In contrast to suspects screening, non-target (unknown) screening in a strict sense starts without any a priori information on the compounds to be detected. Many studies in the literature thus fall in between these two categories, as in systems with well-controlled boundary conditions such 
Fig. 3 a Plot of nominal $\mathrm{m} / \mathrm{z}$ vs. mass defect of all matrix ions observed in two retention time (Rt) windows of a full-scan HRMS chromatogram at a resolution of $60,000(\mathrm{~m} / \mathrm{z} 150-450)$ from a background soil extract. b Extracted ion chromatograms ( $R=60,000$, mass accuracy window $5 \mathrm{ppm}$ ) of the herbicide linuron spiked into a background soil extract $(4 \mathrm{ng} / \mathrm{mL}$, corresponding to $20 \mathrm{pg}$ on column) and of a suspected transformation product of lenacil in a soil extract, both showing a nominal $[\mathrm{M}+\mathrm{H}]^{+}$of $m / z 249$ in electrospray ionization, but a different mass defect. c Distribution of all combinatorial possible even-electron $[\mathrm{M}+\mathrm{H}]^{+}$ions of a nominal $\mathrm{m} / \mathrm{z}$ of 249 containing 6-20 C, 1-40 H, 0-8 O, and $0-8 \mathrm{~N}$ atoms as a function of mass defect as well as ions of nominal $\mathrm{m} / \mathrm{z} 249$ actually present in the soil extract matrix in two retention time windows. The category's bin sizes on the histogram correspond to a resolving power of 20,000 , showing roughly the number of ions which cannot be separated at that resolving power (note that this is only an illustrative approximation, as the mass defects are more continuously distributed within each bin's range)
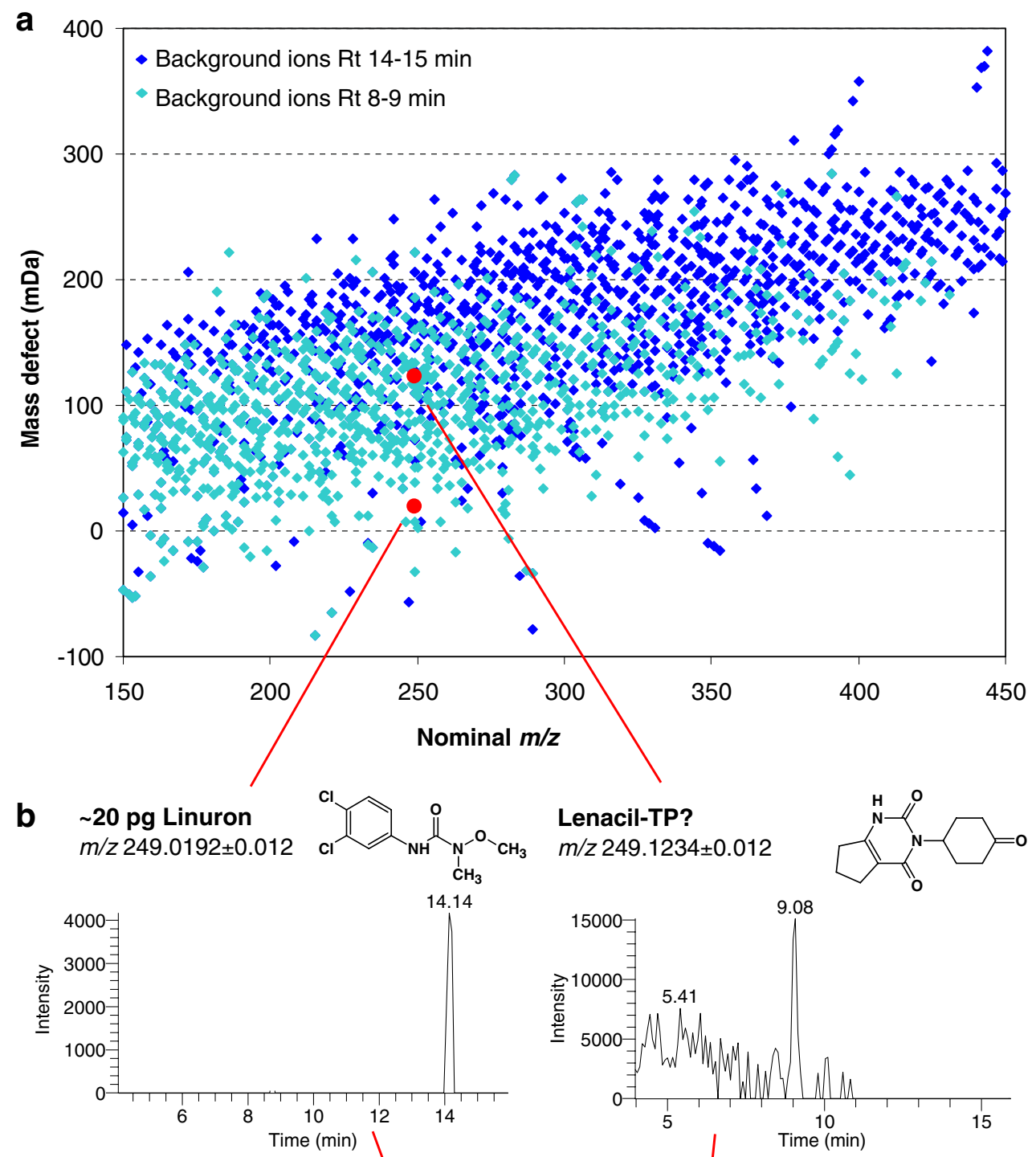

C

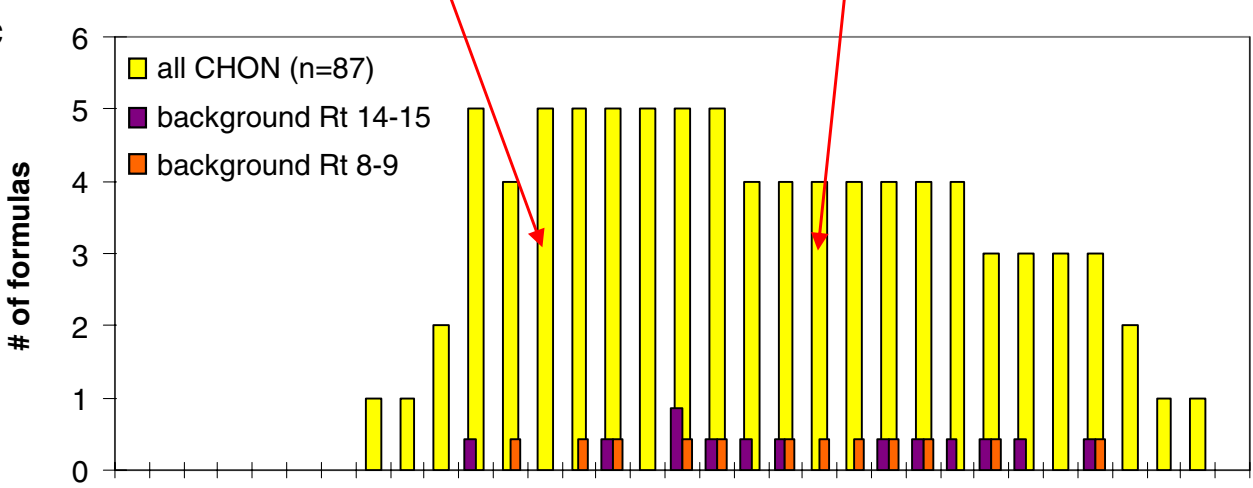

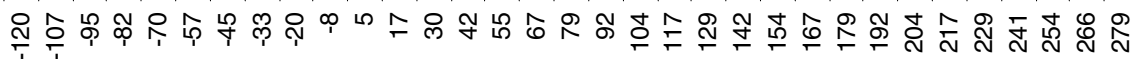

as transformation experiments, e.g., [20-22], the number of chemically meaningful structures which can be assigned to an unknown peak detected is limited to structures showing a close relationship with the parent compound and an adequate control sample or time series is available. For this type of experiment, an identification by HRMS(/MS) alone can often be considered as definitive. A tentative identification of non-target analytes in environmental samples with 
unconstrained boundary conditions is more challenging, and a structure proposition for a peak detected by high resolution MS and MS/MS spectra involves several workintensive data and expert processing steps. Several approaches differing in number and order of the evaluation steps are discussed in the literature [7, 23-26]. Although the described non-target workflows are often focused on one specific evaluation step, the following key features have emerged: (i) an automated peak detection by exact mass filtering from the chromatographic run; (ii) an assignment of an elemental formula to the exact mass of interest; and (iii) a database search of plausible structures for the determined elemental formula (Fig. 1). For an automated compound detection several software packages using different peak detection algorithms are available and usually offered by the MS manufacturer [7]. The capabilities and limitations of the elemental composition prediction from accurate mass measurements by using heuristic rules [19, 27] or profile MS data after peak shape calibration [28] was comprehensively investigated. Searching in large compound databases (e.g., Pubchem) for possible structures of an elemental formula normally results in numerous hits which need to be ranked further by MS/MS data. The search for unknowns in MS/MS or in-source fragment ion libraries is limited to the recorded spectra of reference standards [29], which is not sufficient for a real unknown screening and suffers from limited comparability among instruments. Therefore an in silico strategy for determining unknown chemical structures by matching measured with computational fragmentation spectra of compounds queried from chemical databases seems to be a valuable tool [30]. However, a prediction of product mass spectra yields a large number of possible fragments, of which a rather small number are actually observed.

The results of an unknown screening are briefly demonstrated for a groundwater sample from the Swiss plateau (more details in [31]). After solid-phase extraction (SPE) enrichment, separation on a C-18 column, and ESI in positive ion mode, full-scan MS spectra with a resolving power of 60,000 (at $m / z$ 400) and data-dependent MS/MS spectra of the most intense parent ions with a resolving power of 7,500 were acquired by using an LTQ Orbitrap. The mass accuracy achieved by external mass calibration was less than 5 ppm.

For compound detection by exact mass filtering of the HRMS scan $(m / z \quad 115-1,000)$ the Formulator software (Thermo Scientific, San Jose, USA) was used, which utilizes an algorithm based on a recursive base peak framing, which iteratively groups peaks occurring within the specified $\mathrm{m} / \mathrm{z}$ window of $\pm 5 \mathrm{ppm}$ and a retention time window of $0.5 \mathrm{~min}$. After subtraction of an analytical blank chromatogram 3,800 peaks with a signal-to-noise ratio greater than 5 were obtained. For further evaluation the most intense peak containing a distinct chlorine isotope pattern was selected, showing an $[\mathrm{M}+\mathrm{H}]^{+}$ion at $\mathrm{m} / \mathrm{z}$ 160.0272 $\mathrm{Da}$ and a retention time of $2.2 \mathrm{~min}$. By considering the accurate mass and the isotope pattern an elemental composition $\mathrm{C}_{5} \mathrm{H}_{6} \mathrm{Cl}_{1} \mathrm{~N}_{3} \mathrm{O}_{1}$ could be unambiguously assigned to the uncharged molecule by constraining the atoms to $\mathrm{C}, \mathrm{H}, \mathrm{N}, \mathrm{O}, \mathrm{S}, \mathrm{Cl}$, and $\mathrm{Br}$ for the molecular formula fit. For the molecular formula fit the Seven Golden Rules software tool, which can be downloaded free of charge from the Internet, was applied [27]. About 100 chemical structures were obtained from a search in Pubchem and Scifinder databases for $\mathrm{C}_{5} \mathrm{H}_{6} \mathrm{Cl}_{1} \mathrm{~N}_{3} \mathrm{O}_{1}$. The comparison of the predicted MS/MS spectrum of all database hits using the Mass Frontier software (HighChem, Bratislava, Slovakia) with the measured MS/MS spectrum ranked chloridazon-methyl-desphenyl first in the list of possible structures. The measurement of a reference standard confirmed the peak as chloridazon-methyldesphenyl, one important transformation product of the herbicide chloridazon [32].

As illustrated, the presence of a distinct isotope signal at $\mathrm{M}+2$, as is the case for $\mathrm{Cl}-, \mathrm{Br}-$, and $\mathrm{S}$-containing compounds is important for the elemental composition elucidation. Thus, unknown identification is currently biased to these types of compounds. For other unknown substances containing $\mathrm{C}, \mathrm{H}, \mathrm{N}, \mathrm{O}, \mathrm{F}$, and $\mathrm{P}$ only, which show subtle and low intensity isotope differences both resolving power and spectral accuracy of the MS will determine the performance of the elemental formula fit [19]. Currently, TOF instruments seem to have a somewhat better spectral accuracy $(>1 \%)$ than Orbitrap instruments, exhibiting a spectral error of $3 \%$ and $10 \%$ at a resolving power of 7,500 and 100,000, respectively [28]. Furthermore the MS/MS prediction alone is not sufficient for a successful assignment of a suitable structure to a molecular formula; additional computational approaches are required in this workflow step. The estimation of retention times from estimated physicochemical properties as shown for the suspects screening by Kern et al. [16] or the use of fragment libraries with accurate mass information (not full-scan MS/MS libraries) is likely to improve the match between measured data and candidate structures.

LC-HRMS is an accepted technology for generating meaningful structure suggestions of suspects and unknowns present at low concentrations in environmental samples. One has to bear in mind, however, that an unequivocal identification of trace-level compounds in environmental systems is in most cases not possible by HRMS alone without the application of additional knowledge, complementary techniques, or an authentic reference standard. As a fast evaluation tool for possible candidates, HRMS is ideal when it is combined subsequently with a powerful 
structure elucidation technique like NMR [33], although this requires sufficiently high concentrations and often an isolation of the unknown compound. Note that in natural product chemistry typically these two spectroscopic techniques along with further information on functional groups and elemental composition are required for the true identification of a new compound.

\section{Future perspectives}

The preceding sections demonstrate that LC-HRMS opens the possibility to identify polar target, suspect, and nontarget compounds with improved reliability and robustness. Accordingly, within the 2002/657/EC guideline [34] HRMS precursor and product ions $(R>20,000)$ earn 2 and 2.5 identification points, respectively, instead of 1 and 1.5 points for low resolution MS precursor and product ions. However, additional and more precise criteria for the use of mass accuracy and mass resolution have to be implemented here to define clearly the requirements for a reliable confirmation with LC-HRMS technologies [17, 35]. For laboratories which produce legally relevant residue analytical results, this improved confidence will become increasingly important. Therefore it seems likely that despite the higher investment costs as compared with LC-QqQ instruments, LC-HRMS instruments will find their way from research into routine analysis. With regard to environmental monitoring programs, a major advantage of LC-HRMS is the possibility of retrospective analysis of full-scan data, which enables laboratories to search for "new" contaminants years after data recording. Intelligent strategies allow for combining target analysis and suspects and non-target screening into the same analytical run, including the recording of product ion spectra for target and suspected compounds and intense unknown peaks by data-dependent MS/MS analysis.

Emerging LC-MS ionization techniques such as APPI could possibly extend the analytical window towards less polar compounds in the future. However, the application of APPI for the screening of suspects and unknowns is complicated by the fact that a range of different ions from one compound might be formed (molecule ion, (de) protonated molecule ion, adducts ions), which depends strongly on ionization conditions and is not uniform among compounds of different properties [36].

For reliable formula assignment, an $R>60,000$ is recommended and the accuracy of the isotope intensities must be excellent to allow also elemental formula fits for substances without highly characteristic isotope pattern (consisting solely of $\mathrm{C}, \mathrm{H}, \mathrm{O}, \mathrm{N}, \mathrm{F}, \mathrm{P}$ ) with distinct isotope patterns. So far the assignment of the most probable chemical structure to an elemental formula is the most challenging step in the non-target screening workflow. To increase the success rate of this step, existing approaches have to be optimized like the ability to model MS/MS spectra including product ion intensities. Furthermore, quantitative structure-property relationships could be used for an improved estimation of chromatographic retention times and ionization efficiencies to support a tentative identification. The study by Chalcraft et al. [37] suggests furthermore that with a refined computational prediction of ionization efficiencies from chemical structure or available physicochemical parameters some progress could be made towards a (semi)quantitative analysis of unknowns and suspects without reference standards.

To enable a non-target screening of environmental samples with several thousand peaks within a reasonable time frame, advanced software solutions are needed with capabilities for automated batch processing and fast database queries. At the moment, integration within handy workflows of software packages is mainly available in the field of metabolomics, which is only partly applicable to environmental samples.

Most probably, the impact of LC-HRMS in environmental analysis will increase within the coming years and this technology will make the environment more transparent with regard to the occurrence and fate of polar micropollutants.

Acknowledgments We thank Thomas Bucheli, Andreas Gerecke, Damian Helbling, and two anonymous reviewers for helpful comments concerning this manuscript.

\section{References}

1. Celiz MD, Tso J, Aga DS (2009) Environ Toxicol Chem 28:24732484

2. Petrovic M, Barceló D (2006) J Mass Spectrom 41:1259-1267

3. Kellmann M, Muenster H, Zomer P, Mol H (2009) J Am Soc Mass Spectrom 20:1464-1476

4. Kosjek T, Heath E, Petrovic M, Barceló D (2007) Trends Anal Chem 26:1076-1085

5. Hernández F, Pozo OJ, Sancho JV, Lopez FJ, Marin JM, Ibáñez M (2005) Trends Anal Chem 24:596-612

6. Pozo ÓJ, Sancho JV, Ibáñez M, Hernández F, Niessen WMA (2006) Trends Anal Chem 25:1030-1042

7. Hogenboom AC, van Leerdam JA, de Voogt P (2009) J Chromatogr A 1216:510-519

8. Lim H-K, Chen J, Sensenhauser C, Cook K, Subrahmanyam V (2007) Rapid Commun Mass Spectrom 21:1821-1832

9. Lacorte S, Fernández-Alba AR (2006) Mass Spectrom Rev 25:866-880

10. Sancho JV, Pozo ÓJ, Ibáñez M, Hernández F (2006) Anal Bioanal Chem 386:987-997

11. Martínez Bueno MJ, Agüera A, Gómez MJ, Hernando MD, García-Reyes JF, Fernández-Alba AR (2007) Anal Chem 79:9372-9384

12. Krauss M, Hollender J (2008) Anal Chem 80:834-842

13. van Leerdam JA, Hogenboom AC, van der Kooi MME, de Voogt P (2009) Int J Mass Spectrom 282:99-107 
14. Loos R, Gawlik BM, Boettcher K, Locoro G, Contini S, Bidoglio G (2009) J Chromatogr A 1216:1126-1131

15. Schmid Neset TS, Singer H, Longrée P, Bader H-P, Scheidegger R, Wittmer A, Andersson J (2010) Sci Total Environ (accepted)

16. Kern S, Fenner K, Singer HP, Schwarzenbach RP, Hollender J (2009) Environ Sci Technol 43:7039-7046

17. Nielen MWF, van Engelen MC, Zuiderent R, Ramaker R (2007) Anal Chim Acta 586:122-129

18. Li X, Brownawell BJ (2009) Anal Chem 81:7926-7935

19. Kind T, Fiehn O (2006) BMC Bioinformatics 7:234

20. Thurman EM (2006) J Mass Spectrom 41:1287-1297

21. Ibáñez M, Sancho JV, Pozo ÓJ, Hernández F (2006) Anal Bioanal Chem 384:448-457

22. Harir M, Frommberger M, Gaspar A, Martens D, Kettrup A, El Azzouzi M, Schmitt-Kopplin P (2007) Anal Bioanal Chem 389:1459-1467

23. Ferrer I, Thurman EM (2003) Trends Anal Chem 22:750-756

24. Bobeldijk I, Vissers JPC, Kearney G, Major H, van Leerdam JA (2001) J Chromatogr A 929:63-74

25. Ibáñez M, Sancho JV, Pozo ÓJ, Niessen WMA, Hernández F (2005) Rapid Commun Mass Spectrom 19:169-178

26. Ibáñez M, Sancho JV, Hernández F, McMillan D, Rao R (2008) Trends Anal Chem 27:481-489

27. Kind T, Fiehn O (2007) BMC Bioinformatics 8:105
28. Erve JCL, Gu M, Wang Y, DeMaio W, Talaat RE (2009) J Am Soc Mass Spectrom 20:2058-2069

29. Liao W, Draper WM, Perera SK (2008) Anal Chem 80:77657777

30. Hill DW, Kertesz TM, Fontaine D, Friedman R, Grant DF (2008) Anal Chem 80:5574-5582

31. Hollender J, Singer H, Hernando D, Kosjek T, Heath E (2009) In: Kassinos D, Kümmerer K, Bester K (eds) Xenobiotics in the urban water cycle: mass flows, environmental processes, mitigation and treatment strategies, Springer, Dordrecht, pp 195-211

32. Buttiglieri G, Peschka M, Frömel T, Müller J, Malpei F, Seel P, Knepper TP (2009) Water Res 43:2865-2873

33. Godejohann M, Heintz L, Daolio C, Berset J-D, Muff D (2009) Environ Sci Technol 43:7055-7061

34. European Commision (2002) Commision Decision 2002/657/EC implementing Council Directive 96/23/EC concerning performance of analytical methods and the interpretation of results. Off J Eur Commun L221/8

35. Hernández F, Ibánez M, Sancho JV, Pozo SJ (2004) Anal Chem 76:4349-4357

36. Kauppila TJ, Kuuranne T, Meurer EC, Eberlin MN, Kotiaho T, Kostiainen R (2002) Anal Chem 74:5470-5479

37. Chalcraft KR, Lee R, Mills C, Britz-McKibbin P (2009) Anal Chem 81:2506-2515 